Supporting Information

\title{
White Polymer Light-Emitting Diodes Based on Exciplex Electroluminescence from Polymer Blends and a Single Polymer
}

Junfei Liang, ${ }^{\dagger}$, Sen Zhao, ${ }^{\dagger}+\Varangle$ Xiao-Fang Jiang, ${ }^{\dagger}$ Ting Guo, ${ }^{\dagger}$ Hin-Lap Yip, ${ }^{\dagger}$ Lei Ying, ${ }^{*, \dagger}$ Fei Huang, ${ }^{*, \dagger}$ Wei Yang, ${ }^{\dagger}$ Yong $\mathrm{Cao}^{\dagger}$

$\dagger$ State Key Laboratory of Luminescent Materials and Devices, and Institute of Polymer Optoelectronic Materials and Devices, South China University of Technology, Guangzhou, 510640

\section{Corresponding Authors}

E-mail: msleiying@scut.edu.cn (L. Ying); msfhuang@scut.edu.cn (F. Huang).

Tel: +86 2087114346

Fax: +862087110606

\section{Author Contributions}

$\$$ These authors contributed equally. 


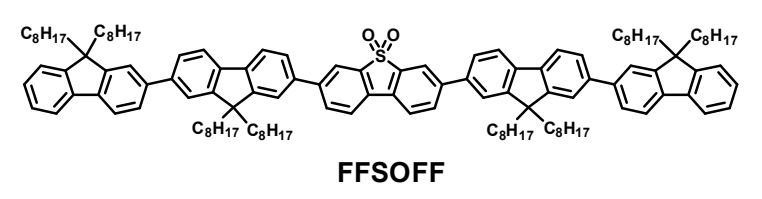

Figure S1. The chemical structure of FFSOFF.

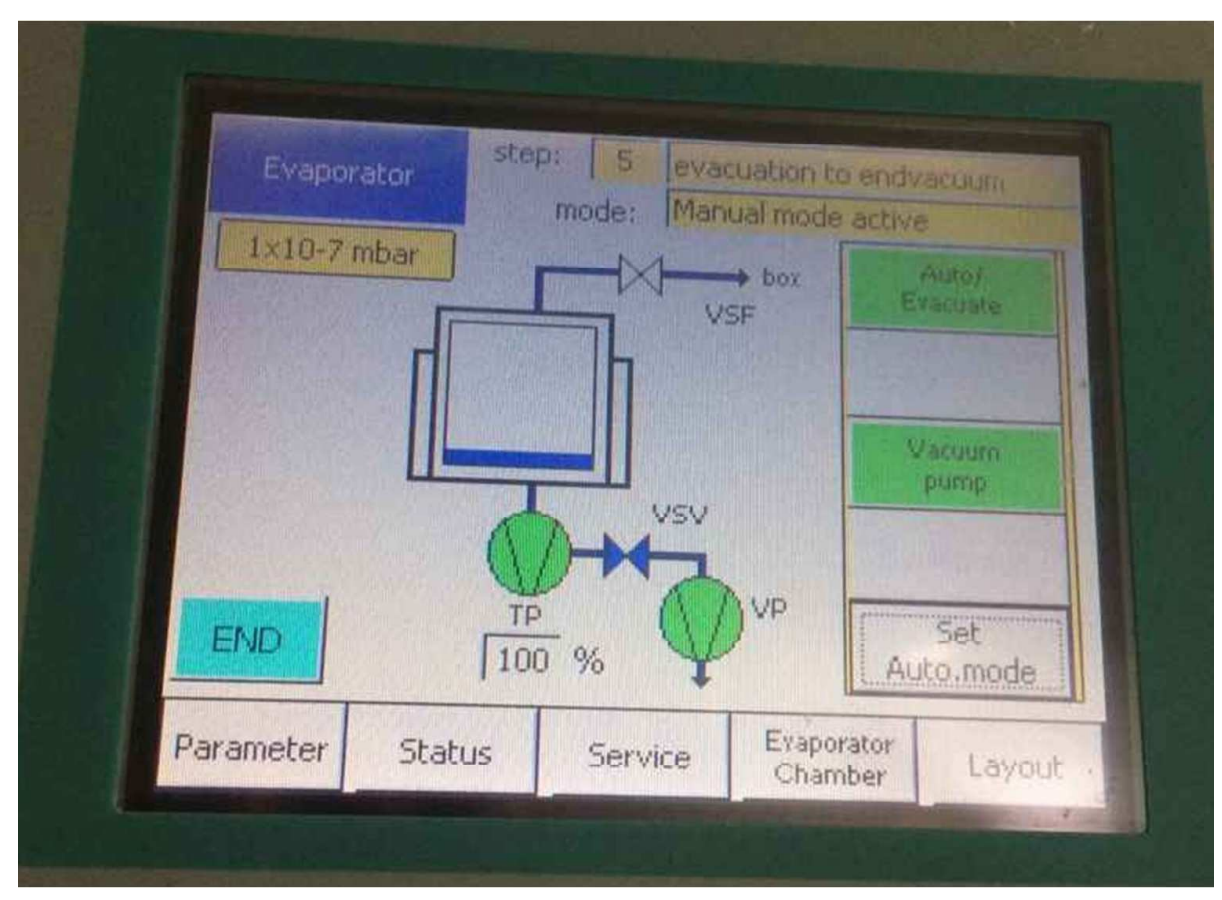

Figure S2. Images of the electronic panel of the vacuum pump system, which comprising of a mechanical pump (EDWARDS: RV12) and a molecular pump (PFEIFFER VACUUM: TC 400 PB, made in Germany in July 2013). 

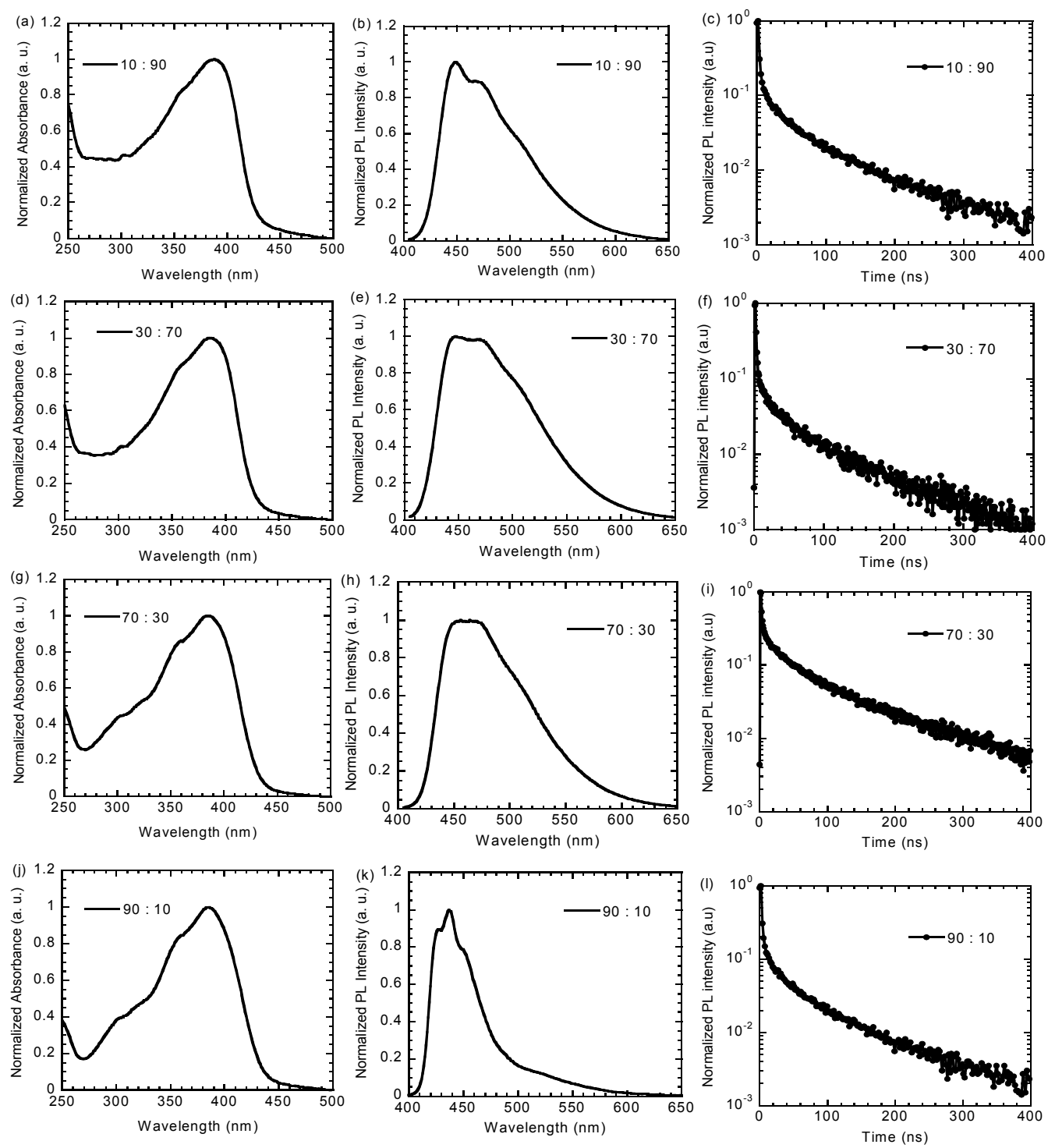

Figure S3. Optophysical performances of films of PF-T25, FFSOFF and PF-T25:FFSOFF (10:90, 30:70, 70:30, 90:10). 

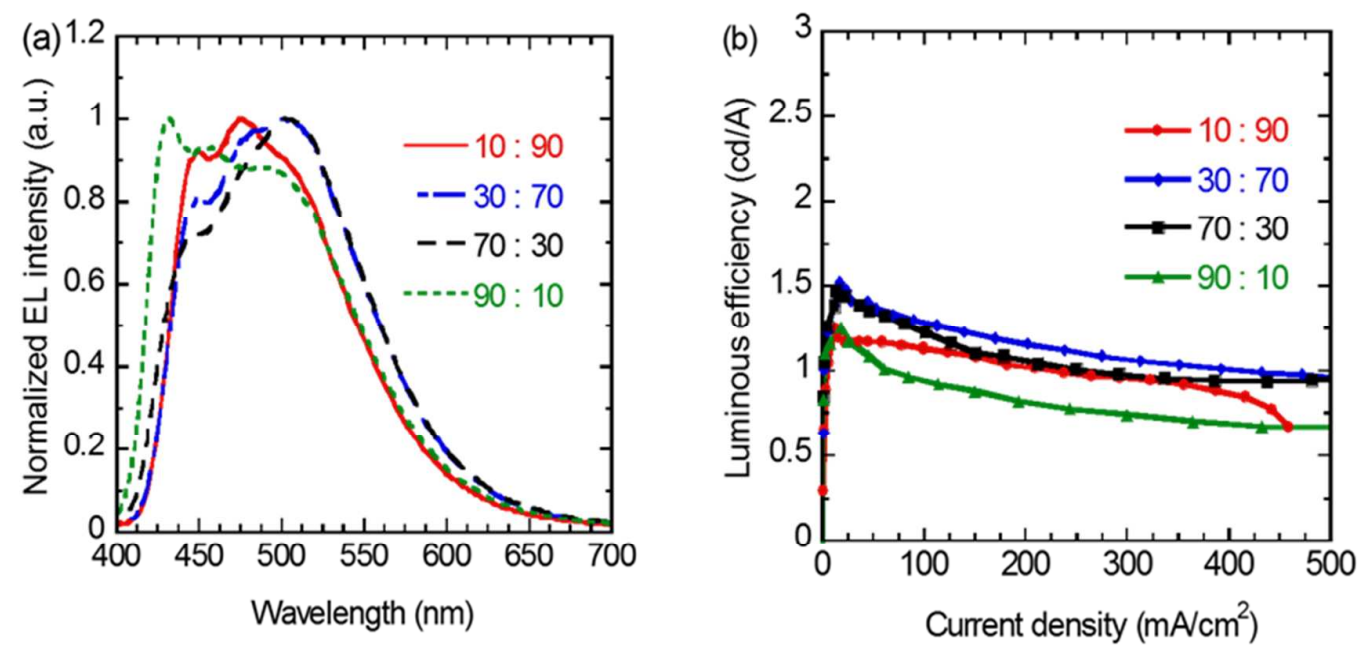

Figure S4. The EL spectra (a) and the luminous efficiency - current density characteristics (b) of the blend exciplex device.

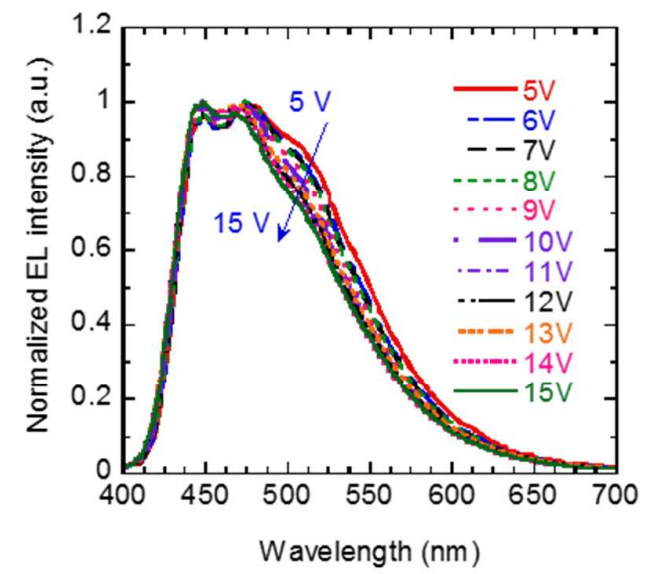

Figure S5. The EL spectra of blend film of PF-T25:FFSOFF $=10: 90$ under different voltages. 


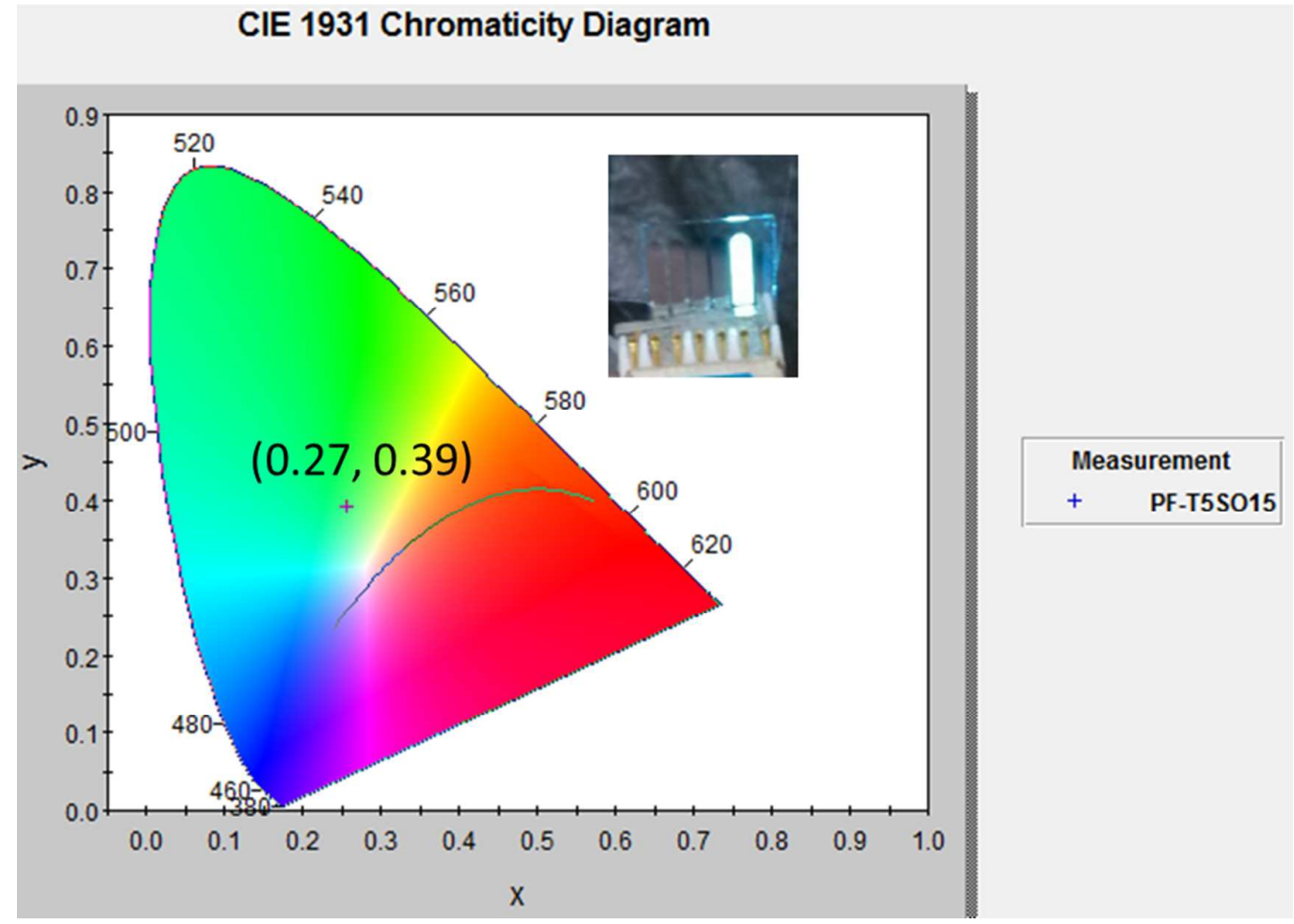

Figure S6. The CIE coordinates of the PLED based on PF-T5SO15 shown in the CIE 1931 chromaticity diagram.

Table S1. The detailed property of fluorescent lifetime for the films of PF-T25, FFSOFF and blend film of PF-T25:FFSOFF $=10: 90,30: 70,70: 30,90: 10$.

\begin{tabular}{llllllll}
\hline Emitter & $\boldsymbol{\tau}(\mathbf{n s})$ & $\mathbf{A}_{\mathbf{1}}$ & $\boldsymbol{\tau}_{\mathbf{1}}(\mathbf{n s})$ & $\mathbf{A 2}$ & $\boldsymbol{\tau}_{\mathbf{2}}(\mathbf{n s})$ & $\mathbf{A}_{\mathbf{3}}$ & $\boldsymbol{\tau}_{\mathbf{3}}(\mathbf{n s})$ \\
\hline $\mathbf{1 0}: \mathbf{9 0}$ & 11.16 & $91.5 \%$ & 3.98 & $8.5 \%$ & 87.35 & - & - \\
$\mathbf{3 0}: \mathbf{7 0}$ & 11.68 & $91.6 \%$ & 3.09 & $8.4 \%$ & 93.49 & - & - \\
$\mathbf{7 0}: \mathbf{3 0}$ & 35.54 & $75.33 \%$ & 4.50 & $13.43 \%$ & 54.89 & $11.24 \%$ & 222.89 \\
$\mathbf{9 0}: \mathbf{1 0}$ & 10.45 & $92.0 \%$ & 3.97 & $8.0 \%$ & 85.17 & & \\
\hline
\end{tabular}


Table S2. The detailed results of the blending exciplex devices.

\begin{tabular}{lllll}
\hline Emitter & $\boldsymbol{V}_{\boldsymbol{t h}}{ }^{\mathbf{a}}$ & $\boldsymbol{L E}_{\max }$ & $\boldsymbol{L}_{\max }$ & $\mathbf{C I E}^{\boldsymbol{b}}$ \\
& {$[\mathbf{V}]$} & {$\left[\mathbf{c d A}^{-1}\right]$} & {$\left[\mathbf{c d m}^{-2}\right]$} & {$[\mathbf{x}, \mathbf{y}]$} \\
\hline PF-T25:FFSOFF $=10: 90$ & 2.9 & 1.25 & 3521 & $(0.20,0.29)$ \\
PF-T25:FFSOFF $=30: 70$ & 2.9 & 1.52 & 5041 & $(0.21,0.33)$ \\
PF-T25:FFSOFF $=70: 30$ & 2.9 & 1.47 & 6557 & $(0.22,0.34)$ \\
PF-T25:FFSOFF $=90: 10$ & 2.9 & 1.26 & 5117 & $(0.20,0.26)$ \\
\hline
\end{tabular}

${ }^{a}$ Turn-on voltage $\left(V_{\mathrm{th}}\right)$ is defined as the voltage at which a luminance of $1 \mathrm{~cd} \mathrm{~m}^{-2}$ is reached;

${ }^{b}$ CIE coordinates are measured at $J=12.5 \mathrm{~mA} \mathrm{~cm}^{-2}$. 